\title{
ZETA AURIGAE TYPE BINARIES
}

\author{
KENNETH G. CARPENTER \\ Laboratory for Astronomy and Solar Physics \\ NASA - Goddard Space Flight Center \\ Greenbelt, MD 20771
}

\begin{abstract}
.
Recent progress in the study of $\zeta$ Aur and VV Cep systems is reviewed, with an emphasis on what has been learned about the extended atmospheres and winds around the cooler components.

Key words: Stars: eclipsing binaries - Stars: chromospheres - stars: late-type - stars: winds
\end{abstract}

\section{Introduction}

$\zeta$ Aur binaries are interacting binary systems with both a hot and a cool component, in which the hot component is eclipsed by the extended atmosphere of the cool component. Their spectra are marvelously complicated: below about $2800 \AA$, the spectra is that of a B-star photosphere on which wind, chromospheric, shock, accretion, and interstellar lines appear superposed. Above $2800 \AA$, the photosphere of the cool star is visible as well, making optical work very entertaining and challenging. In the 'classical' $\zeta$ Aur systems, the cool star is a supergiant, but the class now contains examples where the cool star is a giant. For the purposes of this review, I take this class to include the VV Cep systems, in which the primary is an M-star, as well as the $\zeta$ Aur systems themselves, in which the primary is a $\mathrm{G}$ or $\mathrm{K}$ star.

These stellar systems are of interest for two reasons: 1) for the interactions between the components and 2) for the marvelous opportunity they provide us for the study of the extended atmosphere of the cool components. The visible interactions include shocks and accretion as well as an increased ionization of the cool wind by radiation from the hot star. Eclipses of the hot star by the atmosphere of the cool star allow us to study the height dependence of the thermodynamic parameters (e.g. excitation, ionization, density, temperature, flow and turbulent velocities) of the latter's chromosphere and stellar wind.

In this review I will summarize the most recent work on $\zeta$ Aur systems, concentrating on the very numerous investigations of the extended outer atmospheres of the cool components. The reader is referred to a rich literature of reviews on this topic for further information, particularly on earlier investigations. Some of the major reviews include Schneller (1935), Wellmann (1939), Wilson (1960), Wright (1970), Salhade and Wood (1978), Hack and Stickland (1987), Reimers (1989), and Guinan (1990).

\section{Membership}

Members of the $\zeta$ Aur -type systems which have been well-studied are listed below: 
- Zeta Aurigae Systems (G-K giant/supergiant with hot dwarf companion)

\begin{tabular}{|c|c|c|c|c|}
\hline$=31 \mathrm{Cyg}$ & $\mathrm{K} 4 \mathrm{Ib}$ & $+\mathrm{B} 4 \mathrm{~V}$ & \multicolumn{2}{|c|}{3784 days } \\
\hline$=$ Tau Per & G5 III & $+\mathrm{A} 2 \mathrm{~V}$ & \multicolumn{2}{|c|}{$1515 \mathrm{~d}$} \\
\hline$=32 \mathrm{Cyg}$ & $\mathrm{K} 4 \mathrm{Ib}$ & $+\mathrm{B} 7 \mathrm{~V}$ & \multicolumn{2}{|l|}{$1148 \mathrm{~d}$} \\
\hline$=$ Zeta Aur & K5 Iab & $+\mathrm{B} 7 \mathrm{~V}$ & \multicolumn{2}{|l|}{$972 \mathrm{~d}$} \\
\hline$=$ HR 6902 & G9 II & $+\mathrm{B} 8 \mathrm{~V}$ & \multicolumn{2}{|l|}{$385 \mathrm{~d}$} \\
\hline$=22 \mathrm{Vul}$ & G3 Ib-II & + B9 V & \multicolumn{2}{|l|}{$249 \mathrm{~d}$} \\
\hline$=$ HR 2554 & G6 II & $+\mathrm{A} 0 \mathrm{~V}$ & \multirow{2}{*}{\multicolumn{2}{|c|}{$\begin{array}{c}195 \mathrm{~d} \text { no interaction } \\
96 \mathrm{~d}\end{array}$}} \\
\hline$=\mathrm{AL} \mathrm{Vel}$ & K0 III & $+\mathrm{B} 8 \mathrm{~V}$ & & \\
\hline \multicolumn{5}{|c|}{ VV Cephei Systems } \\
\hline$=\mathrm{KQ} \mathrm{Pup}$ & M2 Iab & \multicolumn{2}{|c|}{$+\mathrm{B} 2 \mathrm{~V}$} & $9750 \mathrm{~d}$ \\
\hline$=\mathrm{VV}$ Cep & M2 Ia & $+\mathrm{B} 9$ & \multirow{2}{*}{$\begin{array}{l}\text { V-IIIe } \\
\text { V }\end{array}$} & $3784 \mathrm{~d}$ \\
\hline$=$ Del Sge & M2 II & $+\mathrm{B} 9$ & & $3720 \mathrm{~d}$ \\
\hline
\end{tabular}

- Weird \& Unique

= Eps Aur F0 Iab + disk ?? 9890 days (primary is lightbulb)

Only true eclipsing systems have been included in the above list. Epsilon Aur is in a separate class all by itself and easily warrants its own review. It will not be considered further in this one.

\section{Interactions}

There are two major types of interactions which occur in $\zeta$ Aur systems: the formation of shocks and accretion disks as the B-star moves supersonically through the cool wind, and the more remote effects on cool star wind due to the presence of the hot star such as gravitational perturbations and ionization by radiation from the B-star.

\subsection{Shocks and Accretion Disks}

Chapman (1981) is credited with the discovery of accretion shocks in the UV. The lines formed include resonance lines of C IV, Si IV, Al II, N V, and excited Fe III (34) and are seen variously as very broad absorption, regular or inverse $\mathrm{P}$ Cyg, or pure emission profiles. The emitting region clearly moves with the B-star. Che-Bohnenstengel and Reimers (1986) demonstrated that the geometric size of the accretion shock is small compared to the size of the circumstellar envelope and can thus be neglected in wind analysis.

Ahmad (1989) has recently shown that 31 Cyg has an intermediate severity shock in comparison with other $\zeta$ Aur systems. He notes that $\zeta$ Aur and 22 Vul have strong reverse $\mathrm{P}$-Cyg profiles in their high-temperature lines and show high-velocity absorption by C IV and N V, while 31 Cyg shows only weak reverse P-Cyg profiles that are visible only at angles near that of the shock cone and absorption in $\mathrm{C} I V$, but not in N V. 32 Cyg shows no reverse P-Cyg profiles and no C. IV/N V absorption. He further notes that the $82 \mathrm{~km} / \mathrm{s}$ Fe II and Mg II absorption components are circumstellar in origin and consistent with the wind terminal velocity of $80 \mathrm{~km} / \mathrm{s}$. 
Broad or double-peaked emission is taken as the possible signature of an accretion disk. The latter has been seen in $\delta$ Sge by Reimers and Schroder (1983), while Che-Bohnenstengel and Reimers (1986) report signs of an accretion disk in $\zeta$ Aur and $\delta$ Sge.

\subsection{Effects on Cool Wind}

More remote effects of the B-star on the wind of cool component include the influence of the gravitational field of the hot star on the latter's wind and flow patterns and perhaps more significantly the ionization of the cool wind by the radiation from the B-star. This radiation creates an H II region around the B-star and inside the cool star envelope and certainly affects mass-loss estimates for ions such as $\mathrm{Fe}, \mathrm{Si}$, etc.

\section{Probing the Winds of Cool Giants and Supergiants in $\zeta$ Aur Systems}

One of the most important characteristics of $\zeta$ Aur systems is that they allow us to probe the height dependence of the thermodynamic parameters of the outer atmospheres of cool, high-luminosity stars. This information can be used to increase our understanding of the structure of these outer atmospheres and to constrain models of chromospheric heating and wind-driving mechanisms. The cool winds and chromospheres are visible through both emission and absorption lines. Hempe and Reimers (1982) summarize the characteristics of the wind lines of Fe II, Si II, Al II, Mg II, S II, and O I: 1) they have P Cyg profiles in all phases, except that they go into pure emission during totality (resonance scattering of B-star photons), 2) Fe II (UV 9) however remains in pure absorption, due to the strong emission from UV 191, and allows a COG analysis during all phases, and 3) chromospheric lines are seen near totality as narrow absorption lines at zero velocity.

UV and optical spectra each have advantages and disadvantages, but provide very nice complementary information, if both are available for the same object. UV spectra suffer minimal contamination by the cool star, have a well-defined continuum over which resonance-scattered wind lines can be easily studied, contain strong lines of all common chromospheric species, and allow an examination of the chromosphere out to 1-2 stellar radii. However, the UV spectrum is very crowded with lines and analysis can be difficult. Bauer and Stencel (1989) provide a very handy atlas of the 31 Cyg UV spectra, complete with line identifications. Optical spectra suffer from a dual continuum, but have much less crowded absorption features, so that weak lines are more easily measured. More neutrals are available and lower layers of the chromosphere can be studied than in the UV. It is especially useful to have the Balmer series observed since that provides critical modeling information on the hydrogen ionization. VV Cep systems are in general more difficult to analyze than $\zeta$ Aur systems since the optical spectrum is more complex and the M-supergiant has strong emissions in the UV. 


\subsection{Results from Curve-of-Growth Techniques}

Computer codes to handle the non-spherically symmetric line transfer needed to model the profiles have been developed by Hempe (1982) and Baade $(1986,1990)$. Hempe used a 2-level escape probability approximation for the source function to get mass-loss rate estimates. Baade (1986) validated these results with a multi-level modification, although neither calculation matches profiles exactly at all phases.

Measurements of the wind velocity, turbulent velocity and mass-loss rate have been published by Che et al. (1983) for $\zeta$ Aur , $31 \mathrm{Cyg}$, and $32 \mathrm{Cyg}$, while Reimers and Che-Bohnenstengel (1986) have measured $v_{\text {win. }}=170 \mathrm{~km} / \mathrm{s}$ for $22 \mathrm{Vul}$. Reimers (1989) summarizes turbulence and wind velocity measurements for $\zeta$ Aur systems and notes that the turbulent velocity is, in general, approximately one-half of the wind velocity. Baade (1990) uses a '2-integral operator' to get a better 32 Cyg model in which $\dot{M}=1.5 \times 10^{-8}$ solar masses $/ \mathrm{yr}, \mathrm{v}_{\text {wind }}=90 \mathrm{~km} / \mathrm{s}$, and $\mathrm{v}_{\text {turb }}=30$ $\mathrm{km} / \mathrm{s}$.

Measurements of the density and wind velocity versus height in $\zeta$ Aur systems have been given by Schroder $(1985,1986)$ and, for 22 Vul, Schroder and Reimers (1989). In the first paper, the density results indicate the observed acceleration takes place at greater heights and is more gradual than would be the case for Alfven-driven winds. The second paper gives equations for these parameters based on a COG analysis, the equation of continuity, and the assumption of a steady wind.

Temperature measurements from observed populations of Fe II levels have been derived by Che-Bohnenstengel (1984) for $32 \mathrm{Cyg}\left(\mathrm{T}_{e}=4800 \mathrm{~K}\right.$ for $\mathbf{n}_{e}=0.01$, $10000 \mathrm{~K}$ for smaller electron densities), and by Reimers and Che-Bohnenstengel (1986) for $22 \mathrm{Vul}(20000-40000 \mathrm{~K})$. Schroder (1986)measured a variation in temperature from 8500 to $11000 \mathrm{~K}$ over the region from $0.2 \mathrm{R}_{*}$ to $0.5 \mathrm{R}_{*}$ and inferred that heating occurs where the wind starts.

\subsection{The Transition from Coronae to Cool Winds}

Schroder (1990) has presented a major study of the physical state of supergiant chromospheres in the region of the HR diagram where the transition from coronae to cool winds occurs. He presents direct evidence for a new type of hot circumstellar matter in HR 6902 and HR 2554 and infers that these are red giant stars with a solar-type 'hot' wind but a 'cool' corona. The latter has transition-region-like temperatures, but is extended like a corona. HR 6902 is to the right of the $\mathrm{Mg}$ II dividing line $(\mathrm{V} / \mathrm{R}=0.75)$, but has a highly ionized, high speed, low mass wind $\left(120 \mathrm{~km} / \mathrm{s}, 82000 \mathrm{~K}, \dot{M}=1.5 \times 10^{-11}\right.$ solar masses/year $)$. It is difficult to know how a star such as this relates to the hybrid single stars, because a major signature of the hybrid phenomenon is the presence of C IV and Si IV emission, which in $\zeta$ Aur systems can be caused by binary interactions. Schroder suggests that the hybrid stars occupy the region between $\mathrm{HR} 6902$ and $22 \mathrm{Vul}$ and have radially-diverted wind regions alternately filled with cool and hot matter. He also identifies a logical sequence, which he believes is an ionization effect, for four critical systems, in terms of distance from the dividing line: $22 \mathrm{Vul}$ (neutral extended chromosphere), 
HR 6902 (cool, inner layer, extended but decreased (by a factor of $10^{3}$ ) Fe II absorption and hot circumstellar matter further out), HR 2554 (no dense inner layer, but extended/decreased Fe II absorption and hot circumstellar matter), and $\tau$ Per (decreased Fe II absorption from a thin, low layer only).

\subsection{Recent Progress in Optical Spectroscopy}

Recently, Griffen et al. (1990) defined a method of spectral subtraction with which they are able to isolate the 3650 - $4650 \AA$ spectra of the B-star and the K-star chromosphere in $\zeta$ Aur for the first time. Using optical spectra from the 1987 eclipse of $\zeta$ Aur and this technique, they identified over 260 absorption features from the chromosphere and produced a model for the geometry of the eclipse. Their results indicate that, if the lower chromosphere rotates rigidly with the supergiant, the rotation period is synchronous with the orbital period.

Using the data and spectral subtraction technique from the Griffen et al. (1990) paper, Schroder et al. (1990) produced an empirical model of the lower chromospheric layers of the $\zeta$ Aur primary. They applied a curve-of-growth analysis of the chromospheric absorption spectrum to derive density vs. height for various atomic species, to estimate the ionization ratios of $\mathrm{Fe}$ II/ $\mathrm{Fe} \mathrm{I}$ and $\mathrm{Mg} \mathrm{II} / \mathrm{Mg} \mathrm{I}$, and to derive the electron density and temperature and hydrogen ionization at $2.3 \times 10^{6}$ and $6.1 \times 10^{6} \mathrm{~km}$. They found $\log \left(\mathrm{N}_{e}\right)=9.6$ and 9.2 , respectively, and $\mathrm{T}_{e}=5500 \mathrm{~K}$ at both heights, while hydrogen was found to be $1.8 \%$ and $5 \%$ ionized at the two heights. These results nicely complement IUE results which apply only to higher layers of the chromosphere.

\subsection{Results from Comparing Synthetic Spectra to Observations}

Eaton has taken another approach to the study of $\zeta$ Aur systems, by attempting to match broad regions of UV and optical observational and synthetic spectra to determine the chromospheric ionizations, column densities, temperatures, and turbulent velocities. Eaton (1988) defines the procedure and applies it to $31 \mathrm{Cyg}$, while Eaton (1991) presents results for $\zeta$ Aur itself. He 'fudges' NLTE effects and uses the LTE Kurucz (1979) model atmosphere program ATLAS to produce the synthetic spectra and derive the thermodynamic parameters. The correct ionizations states are forced by restricting the stages allowed and the correct electron density is forced by altering the effective gravity. Hydrogen ionization is derived from the Balmer lines, while $\mathrm{Ca}$ II is used to infer the calcium ionization and the electron density.

The Lyman-alpha column density is derived assuming hydrogen is mostly neutral and metals mostly ionized. Analysis of Fe II, Cr II, Si II column densities give the same results and also agree within a factor of two with the Schroder (1985) results on $\zeta$ Aur. He points out that the Balmer lines are seen to 100 solar radii, while the UV lines are seen to $200-370$ solar radii. His results are similar to semiempirical models based on emission lines in that the temperature rise is the same, the turbulent velocity is about $2-3 x$ the sound speed, and there is no hydrostatic equilibrium vs. the gas pressure. Eaton also believes that the chromosphere is confined by magnetic fields, supported by the turbulent pressure of Alfven waves and 
that the turbulent pressure is sufficient to support the atmosphere. He finds that the electron density remains constant with height $\left(\approx 10^{9}\right)$ and infers that the ionization of hydrogen must rise with height to maintain the density at a constant value.

For 31 Cyg, Eaton (1988) finds that hydrogen is essentially neutral, while the metals are 10000:1 singly-ionized:neutral. He finds a temperature range versus radius of $5000-14,400 \mathrm{~K}$, with a mass-averaged value of $6400 \mathrm{~K}$, a turbulent velocity of $20-25 \mathrm{~km} / \mathrm{sec}$, and abundances which agree with Luck and Lambert (1985).

\subsection{Semi-Empirical Wind Model}

Kuin and Ahmad (1989) have published a new semi-empirical wind model of $\zeta$ Aur systems based on observations of 31 and $32 \mathrm{Cyg}$ and $22 \mathrm{Vul}$. The velocity function, non-thermal velocity, and upper limits on $\mathrm{N}_{e} / \mathrm{N}_{H}$ are as inferred from observations by Schroder (1985), but the terminal velocity is taken from Ahmad and Stencel (1988). They assume that the energy fluxes are supplied by Alfven waves (since acoustic wave fluxes are too low based on observed line broadening), and a radially symmetric, steady outflow, and then derive constraints on damping length to find $\mathrm{T}_{e}$ and $\mathrm{N}_{e}$. The resulting model describes the supergiant wind $\mathrm{OK}$, including the ionization behavior. They find that they need a lower limit on $\dot{M}$ in order to get a cool wind, i.e. that if there is too little mass, the radiative losses don't balance the heating and the outer atmosphere warms up. Finally, they find that the damping scale length of the waves must be small near the star and larger further out.

\subsection{A First Look at a $\zeta$ Aur System with a Ko III Primary}

Eaton et al. (1990) have taken advantage of the unusual chance, provided by the $\mathrm{AL}$ Vel system, to use the binary technique on a giant (rather than a supergiant) star. The AL Vel system consists of a K0 III primary and a B8 V secondary. The latter allows us to probe the chromosphere out to about 2 stellar radii. Two eclipses in this system have been observed with IUE and although the wind lines are saturated in the inner regions, data on the upper chromosphere $(0.7$ and 2.0 stellar radii) are good. This study indicates that common ions are singly-ionized, except in a region near the B-star where the common ions are doubly-ionized and that the excitation temperature inferred from Fe II lines is in the range from 10000 $15000 \mathrm{~K}$. The presence of high ionization lines during totality imply the existence of an accretion disk or shell. Analysis of the $\mathrm{Mg}$ II P Cyg profiles indicates a wind expansion of about $60 \mathrm{~km} / \mathrm{s}$, while the width of the emission lines implies a corotation of the wind out to $9 \mathrm{~K}$-star radii.

\section{First Interferometry of $\zeta$ Aur Systems}

The first interferometric resolution of $\zeta$ Aur , $31 \mathrm{Cyg}$, and $32 \mathrm{Cyg}$, using the I2T CERGA interferometer in the near-IR has been reported by Benedetto and Ferluga (1990), who give angular diameters of $5.5 \pm 0.3,5.7 \pm 0.6$, and $5.5 \pm 0.5$ milliarcseconds, respectively. Combining these results with eclipse timing and orbital velocity information, they derive radii of $154 \pm 13,169 \pm 20$, and $215 \pm 33$ solar radii, and 
distances of $260 \pm 28,282 \pm 23$, and $364 \pm 55$ parsecs, respectively. Based on these results, they conclude that the companion of $\zeta$ Aur is already evolving to luminosity class IV.

\section{VV Cephei Systems}

The analysis of VV Cephei systems has proceeded more slowly than that of $\zeta$ Aur systems since the spectra of the hot and cool components are more difficult to disentangle, but several interesting papers have appeared in the last several years. Hack, Engin and Yilmaz (1989) have studied IUE data on VV Cep taken over the period from 1978-1984. They report that no interstellar absorption is seen, but derive an $\mathrm{E}(\mathrm{B}-\mathrm{V})=\mathbf{0 . 4 0}$, a value which is larger than that derived from optical data. They estimate the spectral type of the companion as A0 II and report that the UV spectrum is mostly absorption, in contrast to the optical spectrum which has lots of strong emission, although UV emission is seen from N I, O I, Mg II, Fe II, and weakly from Lyman $\alpha$. They present a summary of the UV multiplets seen in the spectrum, but no detailed list of the identified lines.

Recent results from the Goddard High Resolution Spectrograph (GHRS) on Hubble Space Telescope may help to explain the huge difference in O I (UV 2) flux seen from VV Cep and $\alpha$ Ori. Hack and Stickland (1987) have pointed out that it is nearly 3000x brighter in VV Cep than in $\alpha$ Ori. Carpenter et al. (1991) have shown, using GHRS spectra, that the large deficiency in observed O I (UV 2) flux from $\alpha$ Ori, relative to the 'typical' late-type star, is apparently due to strong circumstellar absorption by the $(9,0)$ band of the 4th Positive System of CO. Presumably, the circumstellar environment of VV Cep is much warmer due to the presence of the B-star and much less conducive to the formation of $\mathrm{CO}$.

Altamore et al. (1991) present for the star KQ Pup (M1-2 Iab + Be, 26.7 year period) an atlas of IUE high-dispersion, DAO Coude, and ESO spectra which cover the wavelength regions $1223-3228,3180-5160$, and $6540-6590 \AA$ and state that digitized spectra are available upon request.

Rossi et al. (1991) analyze Coude and CAT CES optical spectra of KQ Pup taken over the period 1969-1984 and compare them with IUE spectra taken in 1979. They note that the optical spectrum is that of an M-star photosphere on top of which is emission from the wind regions ionized by the B-star from the ions [S II], [Cu], [Fe II], Fe II, and [Ni II]. The resonance lines and excited lines of doubly-ionized metals are broad and red-shifted by $37 \mathrm{~km} / \mathrm{s}$, relative to the radial velocity of the star. The authors infer from this that the lines are formed in opaque turbulent matter flowing near and downward to the star. They prepare from these data a model in which the emission comes from a cool wind region ionized by the B-star and from a shock at the interface of the hot and cool winds.

\section{Summary}

The capability which $\zeta$ Aur systems provide us for resolving the structure of the extended atmospheres of cool giant and supergiant stars has been agressively exploited in recent years, both in the UV using IUE (Schroder 1990, Baade 1990, Kuin 
and Almad 1989) and from the ground with more sophisticated analysis of optical data (Griffen et al. 1990, Schroder et al. 1990). Eaton $(1988,1991)$ has used both in comparisons with extensive synthetic spectra in a different, but equally promising approach to the study of these systems. The $\zeta$ Aur class has been expanded to include several systems with a class III giant, and the initial study of one of them (AL Vel) by Eaton et al. (1990) indicates that much can be learned about the atmospheres of giant stars as well using the 'binary technique'. The first interferometric resolution of $\zeta$ Aur systems (Benedetto and Ferluga 1990) has provided us with exciting basic information on them, including radii and distances. Detailed work on the VV Cep system KQ Pup has been pursued by Altamore et al. (1991) and Rossi et al. (1991) using both UV and optical spectra, with the latter providing a model for the origin of emission lines in that system. It is clear we can look forward to many more years of exciting developments in the study of $\zeta$ Aur systems using ground-based data and space-based UV spectra from IUE and HST.

\section{References}

1. Ahmad, I. A. 1989, ApJ, 338, 1011.

2. Ahmad, I. A. and Stencel, R. E. 1988, ApJ, 123, 1234.

3. Altamore, A., Rossi, C., and Viotti, R. 1991, A\&AS, submitted.

4. Baade, R. 1986, A\&A, 154, 145.

5. Baade, R. 1990, in 'Evolution in Astrophysics', ed. E. J. Rolfe, ESA SP-310, p. 65.

6. Bauer, W. H. and Stencel, R. E. 1989, ApJS, 69, 667.

7. Benedetto, G. P. and Ferluga, S. 1990, A\&A, 236, 449.

8. Carpenter, K. G., Robinson, R. D., Ebbets, D. C., Brown, A., and Linsky, J. L. 1991, BAAS, 23, 910.

9. Chapman, R. D. 1981, ApJ, 248, 1043.

10. Che-Bonenstengal, A. 1984, A\&A, 138, 333.

11. Che-Bonenstengal, A. Hempe, K., and Reimers, D. 1983, A\&A, 126, 225.

12. Che-Bonenstengal, A. and Reimers, D. 1986, A\&A, 156, 172.

13. Eaton, J. A. 1988, ApJ, 333, 288.

14. Eaton, J. A. 1991, ApJ, submitted.

15. Eaton, J. A., Kondo, Y., McCluskey, G. F., and Shore, S. 1990, AJ, 100, 799.

16. Griffen, R. E. M., Griffen, R. F., Schroder, K.-P., and Reimers, D. 1990, A\&A, 234, 284.

17. Guinan, E. 1990, in 'Evolution in Astrophysics', ed. E. J. Rolfe, ESA SP-310, p. 74.

18. Hack, M., Engin, S. and Yilmaz, N. 1989, A\&A, 225, 143.

19. Hack, M. and Stickland, D. 1987, in 'Scientific Accomplishments of IUE', ed. Y. Kondo, Reidel, NY, NY, p. 445.

20. Hempe, K. 1982, A\&A, 115, 133.

21. Hempe, K. and Reimers, D. 1982, A\&A, 107, 36.

22. Kuin, N. P. M. and Ahmad, I. A. 1989, ApJ, 344, 856.

23. Kurucz, R. L. 1979, ApJS, 40, 1.

24. Luck, R. E. and Lambert, D. L. 1985, ApJ, 298, 782. 
25. Reimers, D. 1989, in 'FGK Stars and T Tauri Stars', eds. L. Cram and L. Kuhi, NASA SP-502, p. 53.

26. Reimers, D. and Che-Bonenstengal, A. 1986, A\&A, 166, 252.

27. Reimers, D. and Schroder, K.-P. 1983, A\&A, 124, 241.

28. Rossi, C., Altamore, A., Baratta, G. B., Friedjung, M., and Viotti, R. 1991, A\&AS, submitted.

29. Sahade, J. and Wood, F. B. 1978, in 'Interacting Binary Stars', Pergammon, Oxford, p. 121.

30. Schneller, H. 1935, Sterne, 15, 185.

31. Schroder, K.-P. 1985, A\&A, 147, 103.

32. Schroder, K.-P. 1986, A\&A, 170, 70.

33. Schroder, K.-P. 1990, A\&A, 236, 165.

34. Schroder, K.-P. and Reimers, D. 1989, A\&A, 208, 223.

35. Schroder, K.-P., Griffen, R. E. M., and Griffen, R. F. 1990, A\&A, 234, 299.

36. Wellman, P. 1939, Veroff. Univ. Berlin-Babelsberg, 12, No. 4.

37. Wilson, O. C. 1960, in 'Stellar Atmospheres', ed. J. L. Greenstein, Univ. of Chicago press, Chicago, p. 436.

38. Wright, K. O. 1970, Vistas in Astronomy, 12, 147. 